\title{
Application of Conductive Polymer-Based Hydrogel in Multi-robot Balance Control
}

\author{
Tianbo Qiao
}

School of Microelectronics, Xidian University, Xi'an 710071, China

Corresponding Author Email: tianboqiao@stu.xidian.edu.cn

https://doi.org/10.18280/acsm.450205

Received: 25 December 2020

Accepted: 12 February 2021

\section{Keywords:}

hydrogel, conductive polymer, flexible electronic device, central pattern generator $(C P G)$

\begin{abstract}
At present, the flexible electronic materials with both good flexibility and conductivity have become a major development trend of electronic materials in the future. Due to their flexibility in construction, controllable mechanical properties and electrical conductivity, the conductive polymer-based hydrogels $(\mathrm{CPHs})$ are expected to become one of the candidate materials in flexible electronics and other fields, and have received extensive attention from researchers. However, the existing $\mathrm{CPH}$ systems generally have shortcomings such as low mechanical strength and single function. To this end, the authors attempt to construct a hydrogel using polyaniline (PANI) as the conductive component and polyacrylic acid (PAA) as the flexible component through in-situ polymerization and physical blending. The designed PAA/PANI conductive polymerbased hydrogel enjoys ultra-long stretchability and high strength. It can be used in the flexible strain sensors and pressure sensors to detect the step cycle of the multi-legged robot in real time, and adjust their rhythm during the walking gait, thereby achieving the physical balance. This paper provides a new idea for the application of the CPHs, and especially offers a wealth of theoretical foundation and practical experience for the research on its application to the flexible strain sensors.
\end{abstract}

\section{INTRODUCTION}

Following the rapid development of flexible electronics, wearable devices, biomedicine, tissue engineering and other fields, flexible electronic materials with both flexibility and conductivity have become one development trend of electronic materials. Owing to their good mechanical flexibility, controllable electrical conductivity, and diversified preparation strategies, CPHs have the potential to be applied in many fields, and attract the attention of researchers. At present, researchers have done a lot of studies in expanding the types and application fields of conductive polymers. The flexible stress/strain sensor is a kind of device that can convert the mechanical change generated by itself or the magnitude of the external force received into an electrical signal output, and has a function similar to that of human skin. Its main performance parameters include: sensitivity, response speed, load-unload cycle stability [1]. Luo's Research Group [2] designed a chemical/physical double cross-linked double network hydrogel. As shown in Figure 1, Luo's research group firstly grafted polypyrrole (PPy) onto chitosan containing double bonds to prepare chitosan-polypyrrole (CS-PPy). Subsequently, acrylic acid (AA) monomer and N,Nmethylenebisacrylamide (MBAA) crosslinking agent system were polymerized in the presence of CS-PPy and $\mathrm{Fe}^{3+}$, to finally obtain a self-healing, ultra-long stretchable (1500\%) polyacrylic acid/chitosan-polypyrrole (PAA/CS-PPy) hydrogels that can be also used for $3 \mathrm{D}$ printing. Such hydrogel can be $3 \mathrm{D}$ printed to construct a flexible strain sensor monitoring the pulse, respiration, finger joint bending, and biceps movement. The sensor can wirelessly transmit the monitored value through a Bluetooth low energy (BLE) module to the smartphone. In addition, the PAA/CS-PPy hydrogel has a piezoresistive effect. When subjected to vertical pressure, the distance between conductive particles in the network is reduced and the number of conductive paths increases, so that the electrical conductivity of the hydrogel is positively correlated with the pressure.

Hydrogel is a soft and wet functional material composed of a three-dimensional cross-linked network and water. The three-dimensional cross-linked network is usually a chemically or physically cross-linked polymer network [3]. In recent years, extensive attention of the researchers has been paid to the environmentally sensitive hydrogels that respond to $\mathrm{pH}$ [4], temperature [5], light [6], electricity [7], magnetism $[8,9]$, etc., and high-strength hydrogels with excellent mechanical properties such as high strain rate and high tensile strength $[10,11]$. Due to the characteristics of water absorption, biocompatibility, and softness, they are widely used in the fields of biomedicine $[12,13]$, tissue engineering, and flexible devices etc. Electrically conductive hydrogels (ECHs) are an emerging functional material with both electrical conductivity and the soft and wet characteristics of hydrogels. Its conductive components can be conductive polymers, electrolytes or inorganic conductive fillers, such as carbon nanotubes and graphene etc.

For flexible electronic materials, good mechanical properties are essential. However, current conductive hydrogels generally suffer from poor mechanical properties. To solve this problem, the double network structure is currently one of the main construction methods, in which the rigid and flexible polymer networks in the gel are combined through physical interpenetration. Although the doublenetwork structure can enhance the mechanical properties of 
hydrogels to a certain extent, rigid and flexible polymer networks only combined by physical interpenetration have the problems of asynchrony and slippage between networks during deformation. Using the traditional crosslinking agents, the obtained flexible hydrogel network substrate generally has low mechanical strength and poor stretchability, resulting in a limited flexibility for the constructed double network hydrogel.

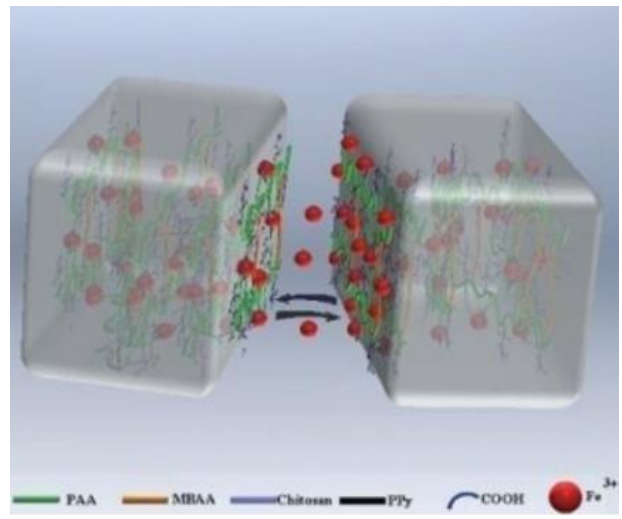

Figure 1. PAA/CS/PPy electrically conductive hydrogel [2]

\section{BIO-ROBOT LEG JOINT MOTION DETECTION}

\subsection{Summary of CPG biological motion control mechanism}

Graham Brown concluded through experiments with cats and guinea pigs that the gait output of the mammalian spinal cord does not require feedback of input signals, and the reflection of the spinal cord can produce rhythmic gait signals. Reflex movement, voluntary movement and rhythmic movement are the three basic types of biological motion [14].

Central Pattern Generator (CPG) is a neural network to generate coordinated patterns of rhythmic activity without any rhythm input from sensory feedback or advanced control centers. They are the basis of many rhythmic behaviors in invertebrates and vertebrates. The CPG model is designed to have multiple levels of abstraction ranging from a detailed biophysical model to a connection model, and then to an abstract system of coupled oscillators [15]. Similarly, a mechanically driven treadmill can induce the normal walking gait of a de-brained cat, and even induce the gait to a trot and gallop when the treadmill accelerates.

The motor system of vertebrates is organized as: the spinal cord CPG is responsible for generating basic rhythmic patterns, and the higher-level centers (motor cortex, cerebellum, and basal ganglia) are adjusting these patterns according to environmental conditions, using the rhythmic activities for coordinated motor control, which is divided into a high-level nerve center and a low-level nerve center, as shown in Figure 2. It can be found from the figure that the distributed organization can reduce: (1) the time delay in the motion control loop (the rhythm is coordinated with the mechanical movement through the short feedback loop of the spinal cord); (2) the dimensionality signal of descent control. Generally, the muscle activity is not required for control signals.

\subsection{Joint movement control}

When using the CPG for the movement control of the bionic animal, it's concluded the law of the hip and knee joints of mammalian limbs in the movement process: during normal walking, the hip joint and knee joint of the same leg have a certain phase relation in a movement cycle; in the supporting phase, the hip joint swings backward, while the knee joint remains basically immobile [16]. The phase relationship between the hip and knee joints for a single leg is shown in Figure 3.

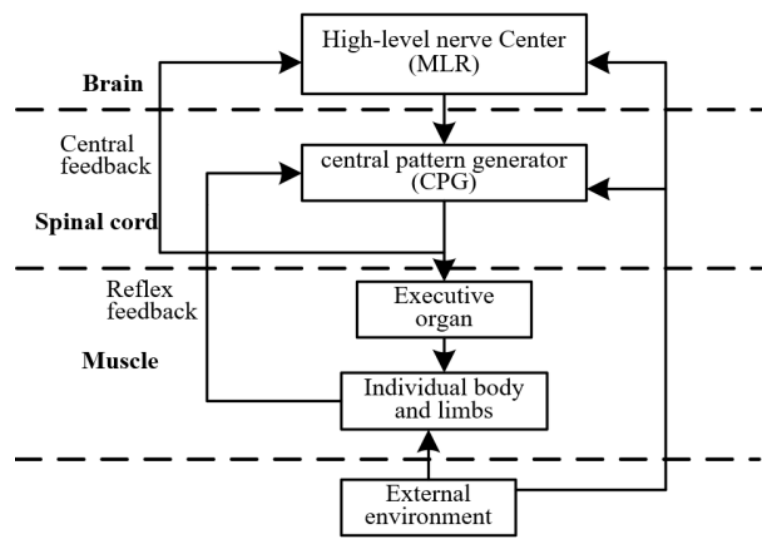

Figure 2. Block diagram of animal rhythmic movement

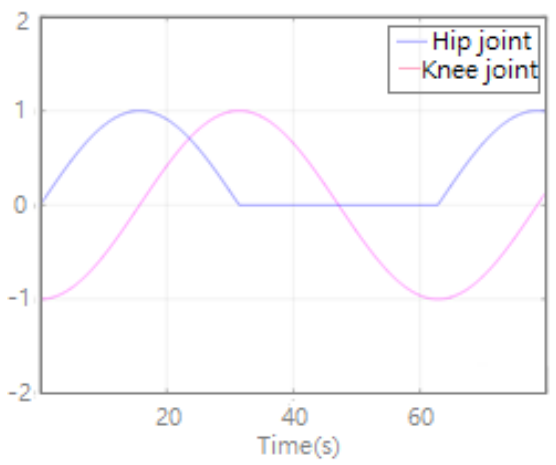

Figure 3. Schematic diagram of the motion relationship between the hip and knee joints of a single leg

Figure 3 shows that there is a certain law of motion between the hip joint and knee joint. Different legs were set according to the joint motion relation, and the corresponding phase angle difference was presented, to ensure that the hexapod robot remains a periodic rhythm and keeps the balance of the body's center of gravity by adjusting the gait, despite of the coupling relationship between the rotation angle variables of each joint. It also prepares for constructing the output signal of the neural network joint controller for the Hopf oscillator in the future.

Combining the characteristics of the hexapod robot, this paper proposes a four-unit control system scheme based on the hierarchical control system structure of the hexapod robot. Figure 4 shows the overall structure of the hexapod robot motion control system, which consists of a central control unit, a multi-axis coordinated motion control unit, a single/multileg execution unit, and a data acquisition and information processing unit.

The central control unit takes the computer as the core to perform decision-making and information processing, and directly controls its lower layer (multi-axis coordinated motion control unit). As the core unit of the control system, it's mainly responsible for the robot's motion mode selection, joint rotation angle calculation, CPG unit oscillation signal generation, sensor feedback information fusion, and timely 
response to the command information input by the operator through RS422 communication, etc. By adjusting the amplitude and phase of the CPG oscillation signal, the central control unit ensures a constant phase difference between neurons, thereby adjusting the robot's movement gait. Also, the sensor provides guidance for the central unit in the decision-making process using the information feedback and ensures the smooth movement of the robot

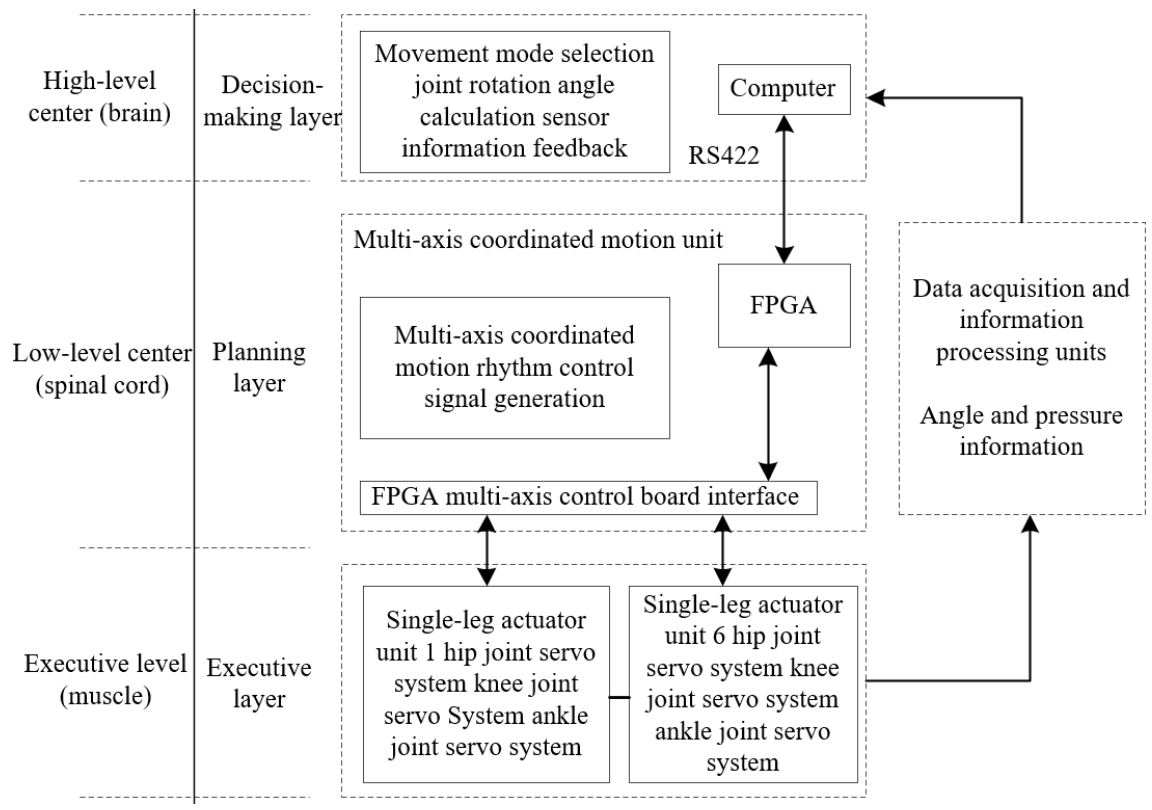

Figure 4. Block diagram of the control system of the hexapod robot

The multi-axis coordinated motion control unit regards the FPGA motion controller as the core, to receive motion commands sent by the central control unit through RS422 communication, and call the corresponding underlying submodule driver according to the motion control instructions, thus driving the coordinated motion of multiple joints. Taking the hip joint of a single leg as the control object of the CPG output signal, the phase differences between the rotation angle waveforms of the hip joints were changed to transform the step sequence of the 6 legs, and then realize different gait output forms. Based on the resolving results of single-leg inverse kinematics, the Knee joints move with the cycle of the hip joint CPG oscillation signal, thereby linking the CPG control signal with the joint motion corner trajectory to realize the gait motion of the robot foot.

The single/multi-leg execution unit is composed of a servo drive system and a servo motor. The servo drive system receives the continuous drive signal sent by the FPGA motion controller, and automatically adjusts it according to its internal current loop and speed loop; also, it feeds the position of the angle sensor back to the motion controller for constructing the position loop of the control system and forming the three-loop control of the servo system, which improves the accuracy and flexibility of single-leg control.

There are pressure sensors and angle sensors in the data acquisition and information processing unit. They are respectively installed at the bottom of the six legs and 18 joints of the hexapod robot body to detect the running status of the robot and the force of each foot and the ground in real time, and process the collected information into digital signals and feed back to the central control unit. Finally, the central control unit decides the robot's movement gait based on the feedback information.

\subsubsection{Selection of CPG oscillator model}

The Hopf oscillator model is a coupled unit. Considering that FPGA hardware realizes parameter adjustment and the model calculation complexity can be converted, the parameters of this mathematical model have clear physical meanings. The setting parameters are less than other common CPG models, and the amplitude and phase of the oscillator are independently adjustable [17]. As shown in Figure 5, the oscillator model consists of an excitatory neuron and an inhibitory neuron, which belongs to the neural network structure. Each neuron has a nonlinear self-feedback effect, and there is a linear coupling between neurons, while the parameter setting process of network structure is not complicated. Thus, external excitation signals were added. The mathematical model of the oscillator is given as:

$$
\dot{X}_{i}=F\left(X_{i}\right)+P=\left[\begin{array}{l}
\lambda\left(K-x^{2}-y^{2}\right) x-w y \\
\lambda\left(K-x^{2}-y^{2}\right) y-w x
\end{array}\right]+\left[\begin{array}{c}
p_{x} \\
p_{y}
\end{array}\right]
$$

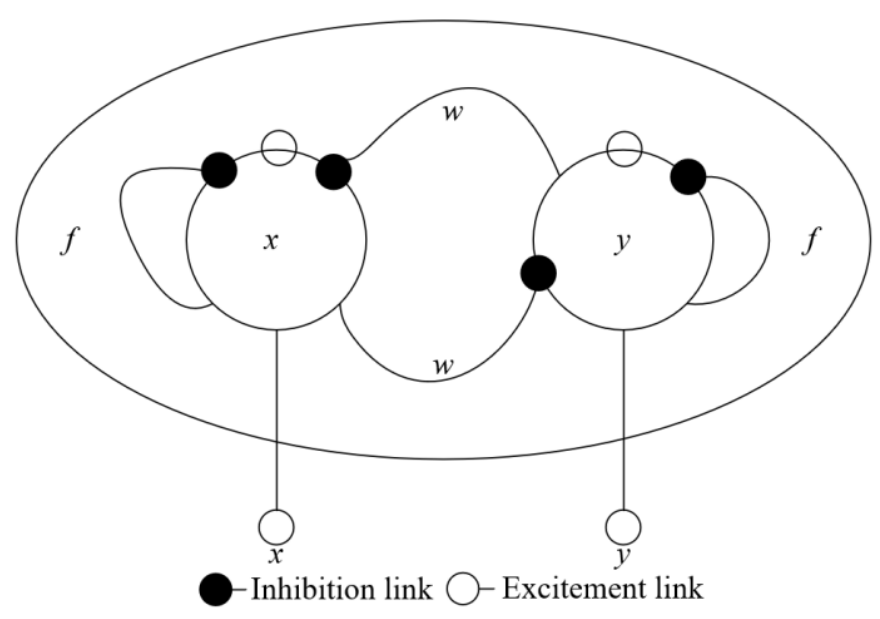

Figure 5. Excitation-inhibition link relation of the oscillator model 
With no external excitation, then $p_{x}=0, p_{y}=0$, and the mathematical model of the oscillator is:

$$
\dot{X}_{i}=F\left(X_{i}\right)=\left[\begin{array}{l}
\lambda\left(K-x^{2}-y^{2}\right) x-\omega y \\
\lambda\left(K-x^{2}-y^{2}\right) y-\omega x
\end{array}\right]
$$

The CPG model based on Hopf coupled oscillation is one of the commonly used neuron models. For the Matsuoka oscillator model [18], the oscillation equation of a single neuron is shown in Eq. (3). Matsuoka stated that the rhythm signal is generated by the simulated of two mutually inhibiting units: extensor (e) and flexor (f).

$$
\left\{\begin{array}{l}
T_{r} \dot{u}+u=s-b v \\
T_{a} \dot{v}+v=y \\
y=g(u-\theta) \\
g(x)=\max (0, x)
\end{array}\right.
$$

where, $T_{r}, T_{a}$ are both the time constants, $b$ is the internal state self-inhibition parameter, that is, the self-inhibition coefficient, $y$ is the output of the neuron, $v$ represents the internal adaptation of the cell, that is, the fatigue degree of the neuron, and $u$ is the membrane voltage of the neuron. The rhythm signal curve was drawn using the software Matlab, as shown in Figure 6. Both the relaxation oscillator based on the VanDerPol model and the oscillator designed based on the Hopf model are nonlinear [19]. Hopf oscillator is a nonlinear, coupled, and simply constructed type. Figure 7 shows the output signal amplitude and frequency output variable curve of the oscillator.

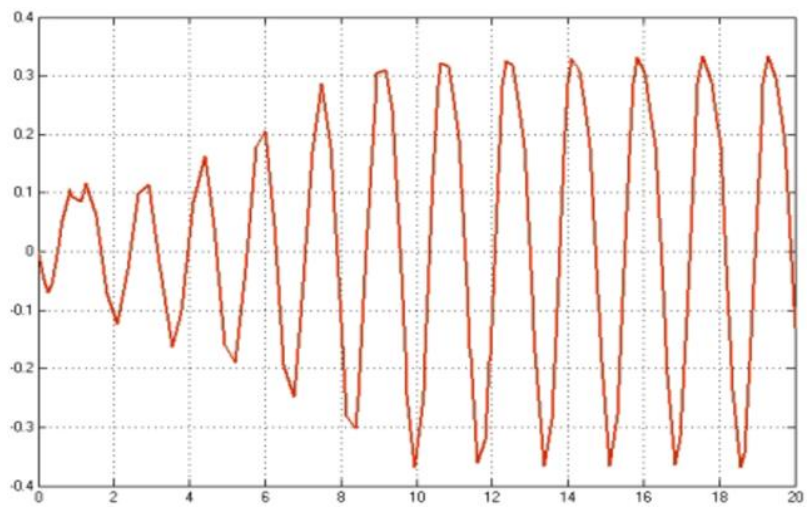

Figure 6. Improved Matsuoka output curve

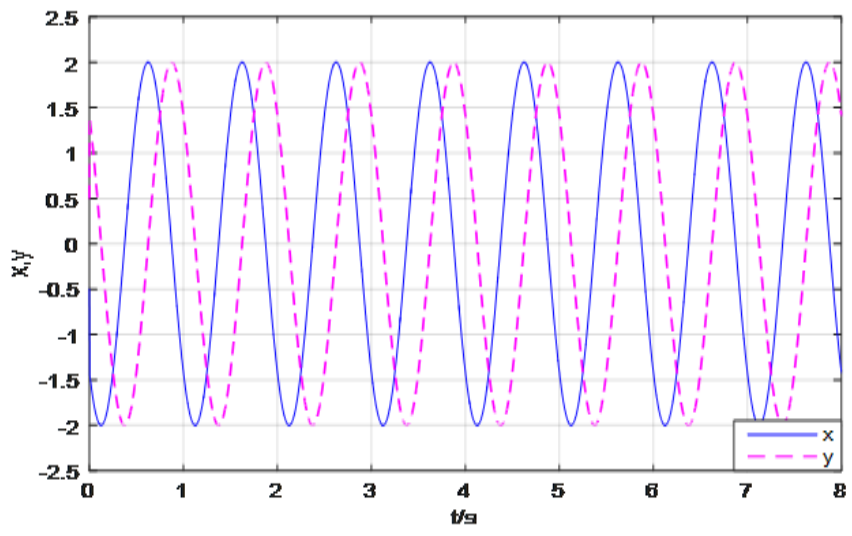

Figure 7. Hopf oscillator output curve

\section{PAA/PANI HYDROGEL BASED STRAIN SENSOR DETECTION}

Based on the reliable sensing performance of the PAA/PANI hydrogel-based strain sensor, this paper further tests its potential for detecting and feeding back the current rhythmic motion of the robot. As shown in Figure 8(a), the PAA/PANI hydrogel-based sensor was installed on the knee joint of the robot. When the joint was cyclically bent (StateA) and released $(\mathrm{State} B)$, the electrical signal $(\Delta R / R 0)$ changed with the bending angle regularly. Figure $8(\mathrm{~b})$ is a test chart of the sensor used to detect joint movement from $0^{\circ}$ to $120^{\circ}$ at the second level. It can be seen from the figure that the relative resistance change $(\Delta R / R 0)$ of the sensor increases with the bending angle of the index finger. Thus, it can detect the movement of the robot foot in real time through the changes of the relative resistance $(\Delta R / R 0)$, laying a foundation for walking balance control.

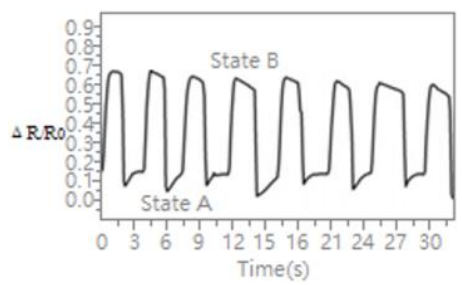

(a) Monitoring of finger bending

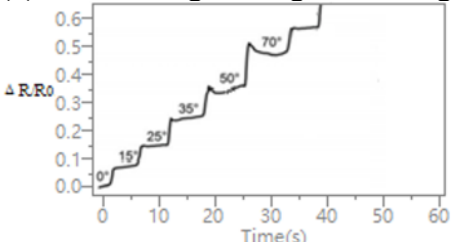

(b) Monitoring of finger bending angles

Figure 8. Human motion monitoring of PAA/PANI hydrogel-based strain sensor

The sensing performance of PAA/PANI hydrogel as a flexible strain sensor was tested in this paper. The test shows that the flexible strain sensor constructed with the hydrogel has an effective strain range of $1130 \%$ and a response time of 0.5 $\mathrm{s}$. It can be used to monitor human movements such as finger joint activities and swallowing etc.

\section{EXPERIMENT AND ANALYSIS OF ANGLE CHANGE DURING ROBOT GAIT WALKING}

The upper computer used the CPG network to generate the control signal of rhythmic joint angle. The oscillator parameter selection was consistent with the setting value selected in Section 3. The control signals were sent to the controller through the wired serial communication module according to the communication protocol. After the controller parses the protocol, they were converted to the angle of each joint, and control the rotation of the motor, thereby realizing the threelegged straight gait of the robot. The three-legged straight gait experiment was conducted as shown in Figure 9 [20].

At the initial time of the experiment, the six legs were in the supporting state; then, the legs 1,3 , and 5 turned to the swing phase, the foot ends swung from back to front along the arc, while the legs 2, 4, and 6 were still in the supporting phase; at about $2 \mathrm{~s}$, the legs 1,3 , and 5 automatically switched to the 
supporting phase, and the legs 2, 4, and 6 became the swing phase, with the feet lifting off the ground and swinging; at about $5 \mathrm{~s}$, the legs 2, 4, and 6 completed the swing and changed to the supporting state, and the foot end was supported from front to back along the ground, so that the center of mass of the robot moved forward, and so on; at $15 \mathrm{~s}$ the actual displacement was about $1.2 \mathrm{~m}$, and the average speed was 0.08 $\mathrm{m} / \mathrm{s}$. Because the discrete point signals are sent by the CPG, the joint angle is actually changing from a discrete angle to another. Due to the limited motor performance and mechanical structure, the robot does not walk at a uniform speed in the actual walking process, but the phase difference between the two groups of legs is stable, with no collision interference, and the phase between the joints meets the gait requirements.

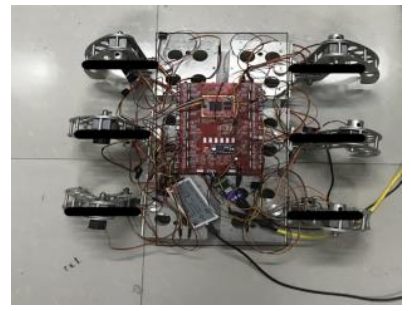

(a)

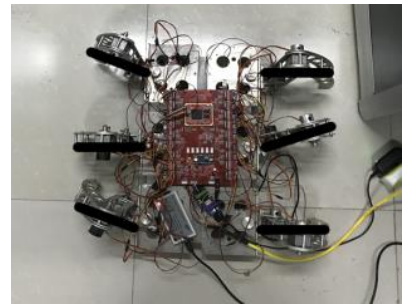

(b)

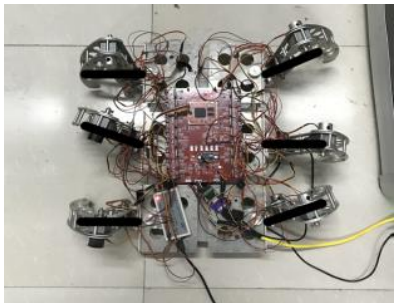

(c)

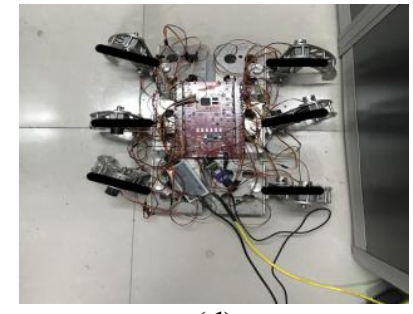

(d)

Figure 9. Pictures of three-legged gaits

In this experiment, the robot was designed to walk in four ways: tripod, tetrapod, wave, and special tripod gait. Figures 10,11 and 12 show the changes in the walking angle of the hexapod robot in each type of gait. The balance of each gait was also analyzed.

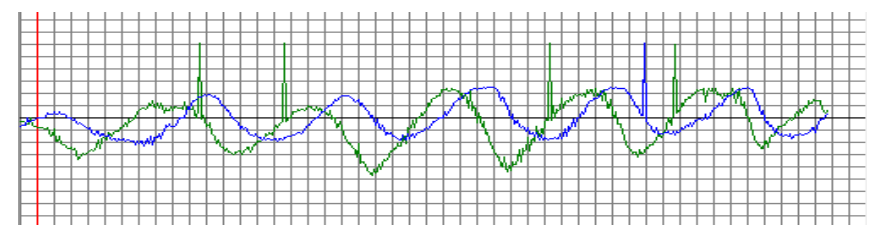

Figure 10. Waveform of angle change in tripod gait

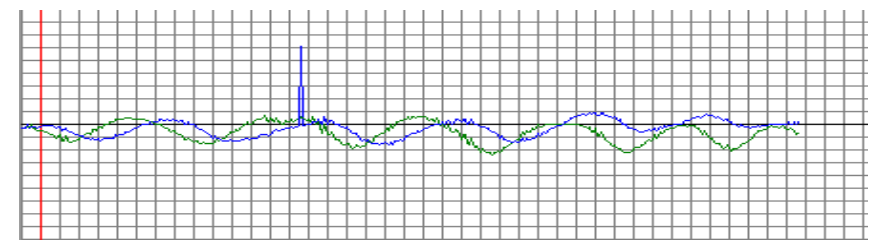

Figure 11. Waveform of angle change in tetrapod gait

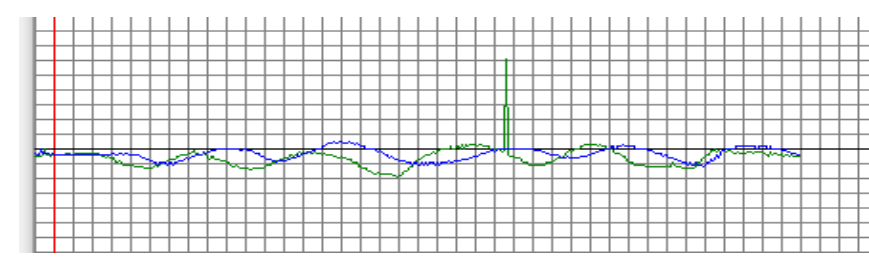

Figure 12. Waveform of angle change in wave gait

It can be seen from the figures that the angle in the tripod gait has the largest change, followed by the tetrapod gait, and the wave gait had the lowest change in the walking angle. Also, the comparison with the alternate periodic sequence of the supporting legs and the swinging legs in the simulated output graphics verifies its hardware system design, realizing the designed walking functions of robot gaits.

In addition, pressure sensors were installed on the foot end of the hexapod robot in the experiment, and the robot was designed to be balanced and inclined postures in all directions through the software design. Then, the experiments were conducted to study the relationship between the change of the knee joint angle and the pressure value of the toe when the posture of the robot changes.

The experimental results found that when the knee joint of one leg raises part of the body of the robot in that direction, the toe pressure value will decrease, while the toe pressure value that is symmetrical to the center will increase, which proves that the body's center of gravity will be closer to the foot with smaller extension angles of knee joint, and the pressure on the toes is inversely proportional to the angle of knee extension.

\section{CONCLUSIONS}

In this paper, the authors developed a conductive polymerbased hydrogel PAA/PANI using F127DA as the PAA hydrogel. For this, the binary synergistic network was taken as the strategy. the co-crosslinking preparation method was adopted. This type of hydrogel has the advantages of long stretchability and conductivity. It can be applied to flexible strain sensors for detecting the moving joints and foot stepping of robots.

\section{REFERENCES}

[1] Chortos, A., Liu, J., Bao, Z. (2016). Pursuing prosthetic electronic skin. Nature Materials, 15(9): 937-950. https://doi.org/10.1038/nmat4671

[2] Darabi, M.A., Khosrozadeh, A., Mbeleck, R., Liu, Y., Chang, Q., Jiang, J., Cai, J., Wang, Q., Luo, G.X., Xing, M. (2017). Skin-inspired multifunctional autonomicintrinsic conductive self-healing hydrogels with pressure sensitivity, stretchability, and 3D printability. Advanced Materials, $29(31)$ :

1700533. https://doi.org/10.1002/adma.201700533

[3] Qiu, Y., Park, K. (2001). Environment-sensitive hydrogels for drug delivery. Advanced Drug Delivery Reviews, 53(3): 321-339. https://doi.org/10.1016/S0169409X(01)00203-4

[4] Yao, L., Krause, S. (2003). Electromechanical responses of strong acid polymer gels in DC electric fields. Macromolecules, 36(6): https://doi.org/10.1021/ma021326q

[5] Yoshida, R., Uchida, K., Kaneko, Y., Sakai, K., Kikuchi, 
A., Sakurai, Y., Okano, T. (1995). Comb-type grafted hydrogels with rapid deswelling response to temperature changes. Nature, 374(6519): 240-242. https://doi.org/10.1038/374240a0

[6] Lo, C. W., Zhu, D., Jiang, H. (2011). An infrared-light responsive graphene-oxide incorporated poly (Nisopropylacrylamide) hydrogel nanocomposite. Soft Matter, $7(12)$ :

5604-5609. https://doi.org/10.1039/C1SM00011J

[7] Ali, A.E.H., Abd El-Rehim, H.A., Hegazy, E.S.A., Ghobashy, M.M. (2006). Synthesis and electrical response of acrylic acid/vinyl sulfonic acid hydrogels prepared by $\gamma$-irradiation. Radiation Physics and Chemistry, 75(9): 1041-1046. https://doi.org/10.1016/j.radphyschem.2005.05.022

[8] Li, Y., Huang, G., Zhang, X., Li, B., Chen, Y., Lu, T., Lu, T.J., Xu, F. (2013). Magnetic hydrogels and their potential biomedical applications. Advanced Functional Materials, 23(6): 660-672. https://doi.org/10.1002/adfm.201201708

[9] Liu, Y.J., Cao, W.T., Ma, M.G., Wan, P. (2017). Ultrasensitive wearable soft strain sensors of conductive, self-healing, and elastic hydrogels with synergistic "soft and hard" hybrid networks. ACS Applied Materials \& Interfaces, $\quad 9(30)$ : 25559-25570. https://doi.org/10.1021/acsami.7b07639

[10] Bilici, C., Can, V., Nöchel, U., Behl, M., Lendlein, A., Okay, O. (2016). Melt-processable shape-memory hydrogels with self-healing ability of high mechanical strength. Macromolecules, 49(19): 7442-7449. https://doi.org/10.1021/acs.macromol.6b01539

[11] Wang, J., Zhang, F., Tsang, W.P., Wan, C., Wu, C. (2017). Fabrication of injectable high strength hydrogel based on 4-arm star PEG for cartilage tissue engineering. Biomaterials, 120: 11-21. https://doi.org/10.1016/j.biomaterials.2016.12.015

[12] Wu, Y., Wang, L., Guo, B., Ma, P.X. (2017). Interwoven aligned conductive nanofiber yarn/hydrogel composite scaffolds for engineered 3D cardiac anisotropy. ACS Nano, 11(6): https://doi.org/10.1021/acsnano.7b01062

[13] Zhao, X., Wu, H., Guo, B., Dong, R., Qiu, Y., Ma, P.X. (2017). Antibacterial anti-oxidant electroactive injectable hydrogel as self-healing wound dressing with hemostasis and adhesiveness for cutaneous wound healing. Biomaterials, 122: 34-47. https://doi.org/10.1016/j.biomaterials.2017.01.011

[14] Bal, C. (2021). Neural coupled central pattern generator based smooth gait transition of a biomimetic hexapod robot. Neurocomputing, 420: 210-226. https://doi.org/10.1016/j.neucom.2020.07.114

[15] Barron-Zambrano, J.H., Torres-Huitzil, C., Girau, B. (2015). Perception-driven adaptive CPG-based locomotion for hexapod robots. Neurocomputing, 170: 63-78. https://doi.org/10.1016/j.neucom.2015.02.087

[16] Rostro-Gonzalez, H., Cerna-Garcia, P.A., TrejoCaballero, G., Garcia-Capulin, C. H., Ibarra-Manzano, M.A., Avina-Cervantes, J.G., Torres-Huitzil, C. (2015). A CPG system based on spiking neurons for hexapod robot locomotion. Neurocomputing, 170: 47-54. https://doi.org/10.1016/j.neucom.2015.03.090

[17] Ijspeert, A.J. (2008). Central pattern generators for locomotion control in animals and robots: A review. Neural Networks, 21(4): 642-653. https://doi.org/10.1016/j.neunet.2008.03.014

[18] Gutierrez-Galan, D., Dominguez-Morales, J.P., PerezPeña, F., Jimenez-Fernandez, A., Linares-Barranco, A. (2020). NeuroPod: A real-time neuromorphic spiking CPG applied to robotics. Neurocomputing, 381: 10-19. https://doi.org/10.1016/j.neucom.2019.11.007

[19] Liu, C., Li, Z., Zhang, C., Yan, Y., Zhang, R. (2019). Gait planning and control for a hexapod robot on uneven terrain based on Markov decision process. In 2019 14th IEEE Conference on Industrial Electronics and Applications (ICIEA), Xi'an, China, pp. 583-586. https://doi.org/10.1109/ICIEA.2019.8834181

[20] Bai, L., Hu, H., Chen, X., Sun, Y., Ma, C., Zhong, Y. (2019). CPG-based gait generation of the curved-leg hexapod robot with smooth gait transition. Sensors, 19(17): 3705. https://doi.org/10.3390/s19173705 